\title{
CORPO REVISOR 2009
}

\author{
Anisio Brasileiro, UFPE \\ Christine Tessele Nodari, UFRGS \\ Eloir de Oliveira Faria, SMTR-RJ \\ Glauco Túlio Pessa Fabbri, EESC-USP \\ Glicério Trichês, UFSC \\ João Alexandre Widmer, EESC-USP \\ Jorge Augusto Pereira Ceratti, UFRGS \\ José Augusto Abreu Sá Fortes, UnB \\ José Tadeu Balbo, EPUSP \\ Laura Maria Goretti da Motta, COPPE/UFRJ \\ Liedi Bariani Bernucci, EPUSP \\ Marcia Valle Real, UFF \\ Marcio de Almeida D'Agosto, COPPE/UFRJ \\ Marcus Vinicius Quintella Cury, CBTU \\ Nelson Maculam Filho, COPPE/UFRJ \\ Paulo César Marques da Silva, UnB \\ Rosa Virginia Ocaña, Universidad Simon Bolívar \\ Suely da Penha Sanches, UFSCar \\ Suely Helena de Araújo Barroso, UFC \\ Vânia Barcellos Gouvêa Campos, IME \\ Washington Peres Núñez, UFRGS
}

